\title{
JOHANN PHILIPP BECKER UND DIE REVOLUTION I848
}

Johann Philipp Becker hat im Jahre I 848 von Biel aus, wo er seit I 838 lebte, ${ }^{1}$ die deutsche Revolution unterstützt. Seine Tätigkeit im Revolutionsjahr I 848 wird in dem folgenden Aufsatz geschildert.

\section{I}

BECKERS BEKENNTNIS FUR EINE DEUTSCHE REPUBLIK I 848

Der Revolution des Jahres I 848 gingen wichtige politische Ereignisse in mehreren Ländern Europas voraus. Die Niederwerfung des Krakauer Aufstandes 1846 und die auf die Einverleibung der Herzogtümer Schleswig-Holstein gerichtete dänische Politik wurden von allen fortschrittlichen Kräften abgelehnt. Der Ausgang des Schweizer Sonderbundkrieges war in den liberalen Kreisen Europas mit Genugtuung aufgenommen worden. ${ }^{2}$

Auch in Deutschland gärte es. Am 27.2.1848 hatten die badischen Abgeordneten Hecker und Mathy in Offenburg Pressefreiheit, Schwurgericht und konstitutionelle Verfassung in allen Einzelstaaten und den Zusammentritt eines Parlaments innerhalb des Bundes gefordert. Acht Tage später verlangte Heinrich von Gagern auf der Heidelberger Tagung, da $\beta$ ein Fürst, ein zentrales Reichsministerium und eine Nationalrepräsentation an die Spitze Deutschlands treten sollten. Während in Heidelberg eine monarchisch-konstitutionelle Führung für das Reich erstrebt wurde, traten Hecker und Struve schon damals für eine Republik ein. ${ }^{3}$

1 Georg Trübner, Der Deutsche Republikaner Johann Philipp Becker als Mitstreiter für die Erringung demokratischer Ziele in der Schweiz, in: International Review of Social History, VI (1961), S. $256-276$.

2 Rudolf Stadelmann, Soziale und politische Geschichte der Revolution I848, München I948, S. 43-44; vgl. z.B.: Das Staats-Lexikon, hrsg. v. Rotteck u. Welcker, Bd. I 2 (Altona I 848), S. 104f.; Werner Näf, Der schweizerische Sonderbundskrieg als Vorspiel der deutschen Revolution von 1848 , Basel r919.

stadelmann, a.a.O., S. 47. 
In der Schweiz verfolgten besonders die vielen politischen Flüchtlinge die Ereignisse in Deutschland mit größtem Interesse. Becker, der damals in Biel wohnte, machte die Stadt zu einem Propagandazentrum für die deutsche Revolution. Er hatte sich selbst am I.3.I 848 aktiv an der Neuenburger Revolution ${ }^{1}$ beteiligt. Dabei war es der republikanischen Partei gelungen, eine neue Verfassung einzuführen, den Kirchenbesitz zu verweltlichen und die Feudallasten zu beseitigen. ${ }^{2}$

Unter Beckers Führung versammelten sich am 26.3.1848 in Biel Delegierte deutscher Vereine in der Schweiz. Die Anwesenden gelobten in Beschlüssen, sich mit allen Mitteln für eine Wiedergeburt Deutschlands einzusetzen. Um dies zu ermöglichen erachtete man eine sofortige Bewaffnung als notwendig und gründete die „Deutsche Legion aus der Schweiz". Sie wurde dem Zentralausschuß der Deutschen aus der Schweiz unterstellt, dessen Sitz Biel war. Der Zentralausschuß sollte die Legion einem zukünftigen vom Volke gewählten Parlament oder einer aus dem Volke hervorgegangenen Behörde zur Verfügung stellen und sich sofort mit der Deutschen Legion in Frankreich in Verbindung setzen. ${ }^{3}$ Der Legion stand ein Kriegstat vor, dem neben Becker noch Daffner, ein ehemaliger Offizier, Schüler, der den ersten deutschen Arbeiterverein in der Schweiz gegründet hatte, ${ }^{4}$ und einige Offiziere angehörten, die sich im Sonderbundkrieg oder bei den Freischarenzügen ausgezeichnet hatten.

In der ersten Sitzung des Kriegsrates unter Beckers Leitung wurde Lommel beauftragt, das "Instruktion" genannte Programm der „Deutschen Legion aus der Schweiz” der entsprechenden vom Volke gewählten Behörde Deutschlands später zur Kenntnisnahme und zur Bestätigung vorzulegen. ${ }^{5}$ In dieser „Instruktion” Beckers ${ }^{6}$ wurden alle Deutschen in der Schweiz aufgefordert, sich sofort in Vereinen zusammenzuschließen. Die Mitglieder dieser Vereine sollten entweder ihre Bereitschaft zeigen, Waffen zu tragen, um auf den ersten Ruf des Vaterlandes die Anordnungen des Zentralausschusses zu befolgen, oder sie sollten sich an der Organisationsarbeit dieser Vereine beteili-

1 N. Rjasanoff, Zur Biographie von Johann Philipp Becker, in: Archiv für die Geschichte des Sozialismus und der Arbeiterbewegung, IV (Igr4), S. 3 I6.

2 T. Curti, Geschichte der Schweiz im XIX. Jahrhundert, Neuenburg o. J., S. 533-534. 3 Aufruf „Deutsche Männerl”, als Einlage zu dem Brief von Joh. Phil. Becker an Karl Mathy aus Biel vom 28.3.1848 (Aufruf datiert Biel, 26.3.1848), in: Aus dem Nachlaß von Karl Mathy, Briefe aus den Jahren I846-I848 mit Erläuterungen, hrsg. von Ludwig Mathy, Leipzig I 898, S. I 2-1 53 (siehe auch S. 154-1 58 ).

- Paul Neitzke, Die deutschen politischen Flüchtlinge in der Schweiz 1848-1849, Diss. Kiel, Charlottenburg 1926, S. 6.

s Neitzke, a.a. O., S. 8; Robert Grimm, Geschichte der Berner Arbeiterbewegung, Band I, Bern 1913, S. 22 r.

- Die -Evolution, ein politisches Wochenblatt, hrsg. von Joh. Phil. Becker, Biel i849, No. II, S. 3 . 
gen und durch entsprechende Geldspenden ihre Opferbereitschaft für das Vaterland bekunden. Die zwanzig von Becker aufgestellten Punkte der „Instruktion” verraten eine gewisse Sachkenntnis des Verfassers auf militärischem Gebiete. So gibt Becker genaue Anweisungen über die Bewaffnung eines Wehrmannes. Wir lesen, wie sich die Bataillone aus je s Kompagnien zu je roo Mann zusammensetzen sollen, wie die Wahl der Kompagniechefs durch Wehrmänner zu erfolgen habe und andererseits die höheren Stabsoffiziere vom Zentralausschuß zu ernennen seien. Solche Männer, die schon auf dem Gebiete des Kriegswesens über angemessene Kenntnisse verfügten, sollten bevorzugt werden. Weiter solle beachtet werden, daß jeder ehemalige Soldat der Waffengattung zugeteilt werde, in der er ausgebildet sei. Der praktische Pfälzer Becker verlangte von seinen Soldaten neben Waffenübungen auch theoretische Kenntnisse auf dem Gebiete des Kriegswesens. Abschließend wird bemerkt, daß alle diejenigen, die jetzt für die zu schaffende Republik Opfer brächten, später von dieser Republik entsprechend unterstützt werden sollten Alle Adressen und Anfragen seien an Johann Philipp Becker zu richten, der als Präsident des Zentralausschusses der Deutschen Legion aus der Schweiz in Biel diese Instruktion unterzeichnet habe. ${ }^{1}$

Der Eifer,'den Becker in Biel damals entwickelte, war bestimmt auch dadurch erhöht worden, daß aus Baden so günstige Nachrichten eingingen. So hatten auf den Versammlungen in Offenburg, Freiburg und Heidelberg am 19., 26. und 27. März Struves Forderungen nach einer deutschen Republik immer mehr an Bedeutung gewonnen. ${ }^{2}$ In einem Brief vom 28.3.1948 an seinen Freund Karl Mathy sprach sich Becker rückhaltlos für die zukünftige deutsche Republik aus:

„Nun ist endlich die Zeit gekommen, in der es eine Freude ist, zu leben. Besonders haben nun Dich die Ereignisse und Entwicklungen auf einem Posten angetroffen, wo Du mit dem ganzen Gewichte Deiner schönen Kräfte mit den Ausschlag gebend dem Vaterlande große Dienste leisten kannst. Du wirst aber auch wie ich nicht ohne Besorgnis sein über die Gestaltung der Dinge in Deutschland, daß nämlich durch Halbheit und Feigheit der meisten deutschen dermaligen Wortführer, die, freilich nur auf den tatkraftlosen Philister gestützt, der republikanischen Bewegung entgegenarbeiten, nur durch eine blutige Revolution die deutsche Republik erlangt werden könne.

Auf eine Republik muß es hinausgehen, sonst gebe ich auf die ganze Bescherung nichts.

1 Die -Evolution, No. I I, S. 3-4.

Stadelmann, a. a. O., S. 49. 
Meine jüngsten Briefe nach Deutschland waren deshalb alle daraufhin gerichtet, daß man sich zuerst der Hofräte, Professoren und Blätter wie die „Deutsche Zeitung” entledige, denn ohne diese Vermittler können die Fürsten gar nicht mehr existieren, wenn jene fort sind, gehen diese ohne weiteres zum Teufel, und die Republik macht sich von selber. Es kann wohl in Deutschland keinen vernünftigen Menschen mehr geben, der ohne Heuchelei sich für eine konstitutionelle Monarchie aussprechen kann?

Wenn die von einem deutschen Parlamente proklamierte Republik noch auf Schwierigkeiten und Gefahren stoßen wird, so wird doch die Gefahr und der Sturm weit größer werden im ganzen Lande, der durch Nichtproklamierung der Republik unfehlbar erfolgen wird. Wir Deutsche in der Schweiz rüsten uns unter vielen Begünstigungen der Behörden entschieden für die deutsche Republik und zwar in Übereinstimmung mit den Deutschen in Frankreich." 1

\section{II}

BECKER UND DER BADISCHE AUFSTAND IM FRÜHJAHR I 848

Die emsige Tätigkeit Beckers in Biel als Präsident des Zentralausschusses der Deutschen Legion aus der Schweiz erweckte den Eindruck, als ob von der Schweiz aus mit einem baldigen Einfall in badisches Gebiet zu rechnen sei. Das Treiben Beckers konnte den Schweizer Behörden, die auf strenge Neutralität achteten, nicht gleichgültig sein. Sie beschuldigten ihn des öfteren, mit seinen verschiedenen Rundschreiben die Neutralität der Schweiz verletzt zu haben. Am 31. 3. 1848 richtete Becker als Präsident des Zentralausschusses an das Regierungsstatthalteramt in Biel ein Schreiben, in dem er diesen Vorwurf zurückwies und ausdrücklich darauf aufmerksam machte, daß seine Legion nur dem Rufe einer aus dem Volkswillen in Deutschland hervorgegangenen Behörde gegen reaktionäre Bestrebungen folgen werde. ${ }^{2}$ In einem Schreiben vom 1.4.I 848 erklärte die Regierung von Bern erneut, daß sie keine fremde Propaganda im Lande dulde. Von dem Regierungsrat in Bern wurde am 5.4.1848 die Bildung militärischer Einheiten, die das Ziel verfolgten, sich in die

\footnotetext{
1 Johann Philipp Becker, Brief an K. Mathy vom 28.3.1848, in: Aus dem Nachlaß von Karl Mathy, S. I II-I 52.

2 Flüchtlinge, Justiz I 848-95, Kasten 4I, Schreiben des Zentralausschusses des deutschen Vereins in der Schweiz an das Regierungsstatthalteramt in Biel vom 31.3.1848, im Bundesarchiv Bern.
} 
politischen Verhältnisse anderer Staaten einzumischen, untersagt. ${ }^{1}$

Auch die Behörden Süddeutschlands rechneten seit Mitte März in verstärktem Maße mit einem Einfall deutscher Republikaner. Dies geht aus einem sehr aufschlußreichen Brief Beckers an Mathy vom 6.4.I848 hervor, ${ }^{2}$ in dem Becker wiederum seine Meinung unterstreicht, daß nur durch die Schaffung der deutschen Republik die großen Schicksalsfragen des deutschen Volkes gelöst werden können. Einen Einfall deutscher Republikaner erwartete man aber nicht nur aus der Schweiz, sondern besonders aus Frankreich. Dort bestand die aus 800 Arbeitern sich zusammensetzende deutsch-demokratische Gesellschaft, deren Leiter der Dichter Georg Herwegh war. Dieser war trotz hoher charakterlicher Eigenschaften als Führer der Legion denkbar ungeeignet. Seinem Plan begegnete man vielerorts mit Skepsis. Hecker traute der Legion nicht viel zu, weil sie meist aus Franzosen bestand. ${ }^{3}$ Der spätere Fehlschlag des Zuges zeigt nur zu deutlich, wie berechtigt die Kritik war.

Zunächst war geplant, daß die Legion Herweghs sich nach ihrer Ankunft in Straßburg mit Heckers Truppen vereinigen sollte. Die Verhandlungen mit Hecker übernahm Frau Emma Herwegh. ${ }^{4}$ Hecker schickte Frau Herwegh zu Weishaar von Lottstetten, der sich am I7.4. I848 mit seinen Leuten in Bewegung setzen sollte, und zu dem Wirt M. in G., der ebenfalls über mehrere hundert Mann verfügte. Außerdem verwies Hecker Frau Herwegh an Becker. Mit Becker, der an der Spitze der „Deutschen Legion aus der Schweiz” stand, sollte Frau Herwegh vereinbaren, daß dieser sich ohne Zögern den beiden anderen Korps anschlösse, um mit ihnen gemeinsam Herweghs Rheinübergang zu ermöglichen. Für uns ist wichtig, daß Frau Herwegh eine Unterredung mit Becker hatte. Wo und wann sie stattfand, wird in dem sonst recht anschaulichen Bericht leider nicht gesagt. Wir erfahren nur das Ergebnis. Becker und M. versprachen, ihre Truppen jederzeit für den Abmarsch bereitzuhalten und nach Vereinigung der drei Korps Depeschen an Herwegh zu schicken und den Ort des Übergangs zu bestimmen.

Beckers Truppen zählten nicht mehr als 400 Mann, allerdings soll es sich um recht erfahrene Verbände gehandelt haben. ${ }^{5} \mathrm{Zu}$ einem

1 Neitzke, a. a. O., S. 12-13.

2 J. Ph. Becker im Namen des Zentralausschusses der Deutschen Legion aus der Schweiz, Brief aus Biel vom 6.4.1848, Io Uhr morgens, in: Aus dem Nachlaß von Karl Mathy, S. 172-174.

s Otto von Corvin, Ein Leben voller Abenteuer, Frankfurt 1924, 2. Band, S. 440.

- [Emma Herwegh,] Zur Geschichte der deutschen demokratischen Legion aus Paris, Von einer Hochverrätherin, Grünberg I849, S. 21.

${ }^{5}$ A. a. O., S. 23-24. 
Einsatz kam Becker jedoch mit seinen Truppen nicht. Als sein Abgesandter Lommel in Straßburg die Mitteilung erhielt, Becker möge aus seinen Truppen zwei Regimenter bilden, das eine unter seiner eigenen, das andere unter Löwenfels' Führung, ging Becker auf diesen Vorschlag gar nicht ein. Sicher hatte er erwartet, daß man ihm die alleinige Führung anvertrauen werde. Wenn Corvin in diesem Verhalten auch eine gewisse Arroganz Beckers sieht, ${ }^{1}$ so haben die militärischen Ereignisse bis zur Niederlage der Truppen Herweghs bestätigt, was Becker schon gleich am Anfang vermutete, daß es nämlich mit der Bewaffnung und Ausbildung der Legion Herweghs schlecht bestellt war. Wenn Becker das Kommando nicht mit Löwenfels teilen wollte, so kann man dies dem erfahreneren Mann nicht verübeln. Hier hätte, wenn überhaupt etwas zu erreichen war, nur eine sehr straffe Führung, die in einer Hand lag, etwas ausrichten können. Dies hatte Becker erkannt und sich deshalb an den Unternehmen Herweghs nicht beteiligt.

Zwar hielt Becker auch die von Hecker geplante militärische Aktion nicht für erfolgversprechend, ${ }^{2}$ trotzdem wandte sich der Zentralausschuß in Biel nach Bekanntwerden des Heckerschen Unternehmens an verschiedene Lokalvereine und forderte deren Mitglieder auf, sich auf den Weg zu machen. ${ }^{3}$ Becker selbst scheint erst eingegriffen zu haben, als er von Struve einen mit Bleistift geschriebenen Zettel erhielt mit der Bitte, sich an dem geplanten Zug zu beteiligen. ${ }^{4}$

Mit 30 Schützen eilte Becker daraufhin aus Biel herbei, um sich mit den gegen Freiburg vorrückenden Truppen Sigels zu vereinen. ${ }^{5}$ Hecker hatte inzwischen eine Niederlage bei Kandern erlitten. Unterwegs leitete Becker am 20.4. 1848 beim badischen Zollhaus auf der Schusterinsel einen Angriff gegen badische Zöllner. ${ }^{6}$ Als Sigel am gleichen Tage in Todtnau eintraf, erfuhr er, daß Becker inzwischen mit 30 Scharfschützen nach Geschwend gelangt sei. Während Sigel nun 20 Wagen nach Zell schickte, damit sie die Truppen von Doll, Mögling und Bruhn nach Todtnau bringen sollten, erteilte er Becker den Befehl, die Straße von Geschwend nach Blasien gegen die anrückenden Württemberger so lange zu decken, bis die aus Zell erwarteten Truppen zu ihm gestoßen seien. Erst dann sollte Becker

1 Corvin, a. a. O., S. 442.

2 Wilhelm Blos, Vom alten Becker. Zur Erinnerung an einen Veteranen der Arbeiterbewegung, in: Die Neue Zeit, V (1887), S. 299.

3 Justiz, Flüchtlinge 1848 -1 895 , Kasten 68 b, Schreiben des Zentralausschusses der Deutschen Legion aus der Schweiz in Biel an den Lokalverein in Burgdorf vom I 8.4.1 848, im Bundesarchiv Bern.

- Blos, a.a.O., S. 299.

5 Ebenda.

- Paul Siegfried, Basel und der erste badische Aufstand im April 1848, Basel r926, S. 41. 
ihm mit seinen Leuten folgen. Der Marsch Sigels von Todtnau nach Freiburg war äußerst beschwerlich, da er über das verschneite Gebirge hinwegführte. ${ }^{1}$ Am 23.4.1 848 trafen Beckers, Möglings und Bruhns ${ }^{2}$ Truppen in Horben endlich mit denen Sigels, die inzwischen schon ein Gefecht bei Günterstal ${ }^{3}$ bestanden hatten, zusammen. ${ }^{4}$ Mit den ihm verbliebenen 400 Mann begann Sigel am 24.4. I 848 (Ostermontag) seinen Angriff auf Freiburg. Diese Stadt beherrschten eine gewisse Zeit die Männer der Volksvereine, die beabsichtigten, sie den anrückenden Republikanern zu übergeben. Bald aber wurde Freiburg von drei Seiten aus von Regierungstruppen angegriffen. Sigels Truppen litten unter Munitionsmangel, viele Freischärler flohen und die Schlacht ging für die Republikaner verloren.

Als letzter Abschnitt des ersten badischen Aufstandes gilt die Besetzung der Schusterinsel. Becker gehörte neben Willich, ${ }^{5}$ der den Befehl über die Insel hatte, und Karl Heinzen ${ }^{6}$ zu den bekannteren Revolutionären, die später spöttisch als „Schusterhelden" bezeichnet wurden. Sie besetzten die Insel vom 25.4. bis zum 27.4. I 848.7 Während der größte Teil der Republikaner, die an der dieser Aktion teilgenommen hatten, ins Innere Frankreichs gebracht wurde, entzog sich Johann Philipp Becker der Internierung und begab sich zurück in die Schweiz. Die Franzosen erhoben keinen Einspruch, da er seit 1 5.I.I 847 als Bürger von Biel Schweizer war. ${ }^{8}$

Becker berichtete später nur allgemeines über seine Teilnahme: „Ich konnte nur schnell, mit einem kleinen Teile meiner Mannschaft, durch einen verwegenen Zug die Sigelsche Kolonne erreichen, während der größte Teil später an der Grenze anlangend, durch verschiedene Hindernisse sich nicht mehr mit mir vereinigen konnte. Aber ich bin noch voll freudiger und dankbarer Gefühle über die herzliche und gastfreundliche Aufnahme, die ich mit meiner Mannschaft an allen Orten des badischen Landes gefunden habe. Es war mir dies eine um so wohler tuende

1 Denkwürdigkeiten des Generals Franz Sigel aus den Jahren r 848/49, hrsg. von W. Blos, Mannheim 1902, S. 34.

2 Karl Bruhn an J. Phil. Becker. Brief vom 29.6.1863, im Nachlaß Becker, Internationaal Instituut voor Sociale Geschiedenis Amsterdam (Bruhn äußert hier seine Meinung über die Revolutionskämpfer von 1848 ).

${ }^{3}$ Friedrich Neff, Brief an J. Phil. Becker vom 25. Juli i 848 , im Nachlaß Becker.

- Denkwürdigkeiten des Generals Franz Sigel, S. 36.

${ }_{5}^{5}$ August Willich, Briefe an J. Ph. Becker vom Juni-Oktober 1848 (Willich gibt in diesen Briefen einen Einblick über das Leben seiner Kolonne in Besançon), im Nachlaß Becker. - Uber Beckers Verhältnis zu Heinzen siehe K. Heinzen, Briefe an J. Ph. Becker vom I6.7.1848, 19.7., 22.7., 19.8. u.a., im Nachlaß Becker.

7 Siegfried, a. a. O., S. 69 .

8 A. a. O., S. 70. 
Tatsache, als ich nach der halboffiziellen Lüge - die Deutschen aus der Schweiz werden von der Bevölkerung mit Mistgabeln und Dreschflegeln empfangen werden - wenigstens keinen so brüderlichen Empfang erwarten durfte. Überhaupt war allenthalben, so weit ich kam, die Begeisterung und Hingebung für die republikanische Sache großartig und erhebend."1

Wenn Becker die Gründe für die Niederlage der Republikaner aufzeigt und meint, daß bei Freiburg noch alles an einem Faden gehangen habe, so scheint mir dies genauso übertrieben wie seine Äußerung, daß er den Kampf noch lange hätte fortsetzen können, wenn die an der Grenze stehenden Deutschen aus der Schweiz rechtzeitig zu ihm gestoßen wären. ${ }^{2}$

\section{III}

\section{BECKERS PROPAGANDISTISCHE TÄTIGKEIT BIS ZUM}

\section{BADISCHEN AUFSTAND}

Am 9.5.I 848 verfaßte Becker als Führer der deutschen Wehrmannschaft bei der republikanischen Revolution im badischen Lande eine Zuschrift an den Fünfziger-Ausschuß in Frankfurt am Main. ${ }^{3}$ Der Fünfzigerausschuß bestand bis zum I 8.5.1 848 und löste sich erst vor dem Zusammentritt der Nationalversammlung auf. ${ }^{4}$ Beckers Kritik am Fünfziger-Ausschuß geht besonders darauf zurück, daß diese Körperschaft die Revolution 1848 in Baden bekämpfte. Nach Beckers Meinung haben Vorparlament und Fünfziger-Ausschuß im Verlaufe der deutschen Revolution I 848 die ihnen zustehenden Aufgaben nicht gelöst. Die Handlungen dieser Körperschaften seien nie der Ausdruck des Volkswillens gewesen, beide hätten eine Versöhnungspolitik betrieben, zwischen den Resten der Fürstenherrschaft und der erwachten Volkssouveränität gestanden und deshalb das ,originelle Experiment” gemacht, eine republikanische Monarchie zu schaffen. ${ }^{5}$ Becker aber forderte, daß die Revolution mit aller Konsequenz bis zu Ende durchgeführt werde. Er erstrebte mit seinen Freunden die Errichtung eines Freistaates, wobei er an die Schweiz als Vorbild dachte. ${ }^{6}$

1 J. Ph. Becker, Zuschrift an den Fünziger-Ausschuß in Frankfurt am Main, von J. Ph. Becker als Führer der deutschen Wehrmannschaft bei der republikanischen Revolution im badischen Lande, Biel I 848, S. 7 .

A. a. O., S. 7-8.

$\checkmark$ Ebenda.

- V. Valentin, Geschichte der Deutschen Revolution 1848/49, Berlin 1930-1931, Band I, S. 530-531; hier zusammenfassendes Urteil über den Fünfziger-Ausschuß.

- Becker, Zuschrift an den Fünfziget-Ausschuß, S. 4.

- A. a. O., S. s. 
Mit einem Rundschreiben als Leiter des Zentralausschusses der Deutschen aus der Schweiz an die Lokalvereine vom I I.6. I 848 wollte Becker die alten Fäden zwischen den Deutschen in der Schweiz, die nach dem Fehlschlag des ersten Badischen Aufstandes abgerissen waren, wieder anknüpfen. Er betonte ausdrücklich, daß der Fehlschlag des gerade beendeten Unternehmens den Glauben der Revolutionäre an den Sieg ihrer Sache nicht erschüttert habe. Wenn die Bewegung fehlgeschlagen sei, dann nur deshalb, weil die Organisation und die Bewaffnung schlecht, die Soldaten im Waffenhandwerk zu wenig geschult gewesen seien. Da in Deutschland im Augenblick der Republikanismus immer mehr Anhänger gewänne, erwachse gerade für die Deutschen in der Schweiz die Aufgabe, alles zu tun, um eines Tages die Erwartungen der deutschen Republikaner zu rechtfertigen. Während Becker den Zentralausschuß der Deutschen aus der Schweiz stärken wollte, war Struve der Meinung, daß unter den bestehenden Verhältnissen der Zentralausschuß der Sache der Republik nichts nütze. ${ }^{1}$

Beckers Rundschreiben gipfelt in der Forderung, daß die deutschen Lokalvereine in der Schweiz sich jetzt um eine systematische Bildung und Aufklärung ihrer Landsleute bemühen müßten. Darüber hinaus sollten alle Mitglieder, die nach Deutschland reisten, sich dort ähnlichen Vereinen anschließen, in Orten, wo noch keine beständen, welche gründen, die dann recht bald Verbindung zum Zentralausschuß in Biel aufnehmen sollten. Das Rundschreiben war von Becker als Präsident und Hattemer als Sekretär unterzeichnet. ${ }^{2}$ Beide Männer wurden wegen dieses Aufrufes in Biel vom Regierungsstatthalter verhört. Becker betonte dabei, daß man keine Schilderhebung plane, sondern sich nur bereithalten wolle, wenn das Vaterland riefe. Militärische Übungen hätten nicht stattgefunden. ${ }^{3}$

In Sommer 1848 konzentrierte Becker seine besondere Tätigkeit auf die immer größer werdende Zahl republikanischer Flüchtlinge in der Schweiz." Die Berner Behörden und auch die Schweizer Bevölkerung waren stolz darauf, daß ihre Heimat als Asyl für politisch verfolgte Menschen galt. Die Überwachung der Flüchtlinge wurde deshalb bis zum Ausbruch des 2. Badischen Aufstandes im September

1 Gustav von Struve, Brief an J. Ph. Becker vom 27.6.1848, im Nachlaß Becker.

2 Das Rundschreiben ist abgedruckt in Die -Evolution, No. II, S. 4.

- Schweizerische Nationalzeitung 1848-1850, No. 165, vom I1. 6. 1848, mitgeteilt bei Neitzke, a. a. O., S. 20.

4 Vergleiche dazu Briefe an J. Ph. Becker von Karl Blind (I I.7. 1848), E. Wegen(1.7.1848), H. Birnbaum (2.7.1848), Jakob F. Hoffmann (I 2.7.1848), H. Schwartz (18.7.1848), J. M. Stöhr (2.8.1848), Chr. Haaf (26.9.1848), u.a. Alle Briefe befinden sich im Nachlaß Becker. 
I 848 sehr lässig durchgeführt. Alle Versuche der badischen Regierung zu erreichen, daß die Behörden die Flüchtlinge in der Schweiz internierten, waren im Mai, Juni, Juli I 848 abgewiesen worden. Man betrachtete -lies als einen Eingriff in das auch den Grenzkantonen zustehende Asylrecht. ${ }^{1}$

Wenn Becker trotz der großzügigen Meinung, die die Schweizer Behörden vom Asylrecht hatten, im Juli I 848 vor ein Gericht gestellt wurde, weil er die Deutschen nach dem Birsfelde eingeladen hatte und aus diesem Grunde das schon erwähnte Rundschreiben an die Lokalvereine vom I r.6.1848 im Namen des Bieler Zentralausschusses erlassen hatte, so ist darin ein zusätzlicher Beweis für die besondere politische Aktivität Beckers während der Sommermonate des Jahres I $848 \mathrm{zu}$ erblicken. Becker hatte sich - so wollte man wissen - selbst als „Oberbefehlshaber" für einen neuen Einfall in Deutschland auserkoren. ${ }^{2}$ Obwohl Becker und Lehrer Hattemer, der spätere Mitarbeiter an der "-Evolution", zu einjähriger Kantonsverweisung verurteilt wurden, duldete man in Biel auch weiterhin ihren Aufenthalt. ${ }^{3}$

Als es am 21.9.1 848 zum Struveputsch kam, zeigte Becker allerdings sehr wenig Interesse, sich Struves militärischer Führung anzuvertrauen. Er hatte zu Struve, den er als „militärischen Führer” vom Frühjahr I 848 her kannte, wenig Vertrauen und wollte nicht mit ihm "putschen".4 Struve seinerseits legte großen Wert darauf, mit Becker zusammenzuarbeiten und rechnete fest mit dessen Unterstützung. ${ }^{5}$ Becker gab mit Alfred Michel am 21.9. 1848 einen Aufruf „Deutsche Brüder" heraus, ${ }^{6}$ in dem darauf hingewiesen wurde, daß die republikanische Bewegung in Deutschland den Charakter einer "gewaltig durchgreifenden Revolution" annehmen und das Vaterland deshalb von den Brüdern in der Schweiz bald die entsprechenden Opfer fordern werde:

„Einstweilen rufen wir nur alle republikanisch gesinnten Deutschen in der Schweiz auf die Wache, damit sie gehörig vorbereitet sind, wenn wir sie nach Entwicklungen im Vaterlande heute oder morgen auffordern, dorthin zurückzukehren. Die

1 Paul Schweizer, Geschichte der Schweizerischen Neutralität, Frauenfeld I895, S. 820.

- Bericht des Regierungsdirektors von Freiburg, Frh. von Marschall, an das badische Ministerium des Inneren, 25.10.1 848, Abschrift A Frankfurt, mitgeteilt bei V. Valentin, a. a. O., Band 2, S. 173 .

- Agentenbericht von 29. Juli 1848, Frankfurtur Bundesarchiv, mitgeteilt bei Valentin, a.a.O., Band 2, S. 173.

- Reinhold Ruegg, Aus Briefen an J. Ph. Becker, in: Die Neue Zeit, VI (1888), S. 454.

- Gustar von Struve an J. Ph. Becker, Briefe vom 14.7. und I. 8. I848 im NachlaB Becker.

- J. Ph. Becker und Alfred Michel, Aufruf „Deutsche Brüder”, Biel 21.9.1848, im Nachlaß Becker. 
Mittel, womit man der deutschen Republik zum Siege hilft, kennt ihr."1

Von allen, die nicht in das Vaterland zurückkehrten, wurden angemessene Geldopfer erwartet.

Struve rechnete fest mit der Ausgabe der von Becker geplanten Schuldscheine, die das Unternehmen finanzieren sollten. ${ }^{2}$ Von den Ereignissen gedrängt, begann er jedoch seinen Putsch, bevor die Schuldscheine geliefert werden konnten. ${ }^{3}$ Er schickte Eilboten zu Becker, schilderte ihm das ganze Unternehmen in rosigen Farben und bat dringend um seine Mitwirkung:

„Ich erwarte von Euch und verlange von Euch, daß ihr gleichfalls in das Badische einrückt, alle Eure Berichte nach Lörrach schickt und mit aller Eurer Macht so schnell wie möglich dahin aufbrecht. Sigel wird bei Konstanz, Löwenfels vorläufig in Lörrach, Bruhn in der Nähe von Mühlheim den militärischen Oberbefehl führen."4

Daraufhin eilte Becker mit der „Deutschen Legion aus der Schweiz” an die Grenze, um, wie er in seiner „Erklärung und Aufforderung”, vom 2. I0.I 848 schrieb, etwas Ordnung in die ganze Sache zu bringen und durch sein diszipliniertes Korps dem ganzen einen Schwerpunkt und Halt zu geben. Er kam zu spät. Es war alles schon verloren.

\section{IV}

DIE UMWANDLUNG DES ZENTRALAUSSCHUSSES DER DEUTSCHEN AUS DER SCHWEIZ IN DEN WEHRBUND ,HILF DIR” UNTER BECKERS PRÄSIDENTENSCHAFT

Es war vorgesehen, daß sich die Freischarenzüge der Flüchtlinge aus der Schweiz mit denen der deutschen Vereine aus Frankreich in Hüningen vereinigen sollten. Dort versammelten sich ungefähr 680 Männer. Sie kamen infolge der raschen Niederlage des Struveputsches nicht zum Einsatz. Ehe sie in ihre jeweilige Wahlheimat zurückkehrten, konstituierten sie sich im Einverständnis mit der Gesellschaft

1 Ebenda, siehe dazu auch: Justiz, Flüchtlinge 1848-1895, Kasten 68b, Mappe: Korrespondenz mit den Behörden von Glarus, Schreiben Beckers an den Deutschen Arbeiterverein in Glarus vom 24.9.1848, im Bundesarchiv Bern.

2 Gustav von Struve an J. Ph. Becker, Brief vom 5.9.1848, im Nachlaß Becker.

3 Gustar von Struve an J. Ph. Becker, Brief vom I 8.9.1848, im Nachlaß Becker.

- Gustar von Struve an J. Ph. Becker, Brief vom 21.9.1848, 16 Uhr, im Nachlaß Becker.

$5 \mathrm{~J}$. Ph. Becker, „Erklärung und Aufforderung”, in: Courier d'Alsace, No. 1 22, vom 6.10.1848, im Bundesarchiv Bern, vergleiche dazu: Robert Grimm, a.a.O., S. 227-230; Fr. B. Teuthorn an J. Ph. Becker, Brief vom 28.9.1848; Alfred Michel an J. Ph. Becker, Brief vom 23.9.1848, 8 Uhr früh; beide Briefe im Nachlaß Becker. 
„Hilf Dir”, die unter Beckers Leitung in Biel aus dem Zentralausschuß der Deutschen aus der Schweiz hervorgegangen war, zu dem Wehrbund „Hilf Dir”.

Hauptaufgabe des Wehrbundes war dieRepublikanisierung Deutschlands, heilige Pflicht des Bundes, jeden äußeren Feind der Schweiz zu bekämpfen. ${ }^{2}$ Becker erhielt für drei Monate die Vollmachten über den Bund. Willich wurde militärischer Leiter; Hofstetten bot sich an, im Winter militärische Vorlesungen zu halten. ${ }^{3}$ Man wollte in diesem Bunde alle Kräfte unter einer starken Leitung vereinen und damit verhindern, daß es wieder zu solch desorganisierten Putschen wie dem Struves kam. Becker forderte alle Deutschen im Ausland auf, sich diesem Bunde anzuschließen. ${ }^{4}$ Die Führer im In- und Ausland sollten sich mit ihm und seinen Bevollmächtigten verständigen. ${ }^{5}$

In seiner „Erklärung und Aufforderung” unterstreicht Becker abschließend noch einmal die freiheitlichen Bestrebungen des Wehrbundes, der als ein Bruchteil des großen deutschen Vaterlandes an einer fortschrittlichen Entwicklung in Deutschland mitarbeiten solle. „Über alles geht die Freiheit, durch sie nur kommt die Einheit und bekommt die Nationalität eine vernünftige Geltung: darum vor allem die Vernichtung der Fürstengewalt, den Völkern die Bruderhand und ein Hoch der Deutschen Republik."6

Der Struveputsch und besonders die Schlußworte des Beckerschen Aufrufes gaben den Schweizer Behörden viel zu denken. Waren sie bisher sehr großzügig gegenüber den Flüchtlingen gewesen, so versuchten sie jetzt, Versäumtes nachzuholen. Wenn man von nun an mit einer gewissen Schärfe gegen Flüchtlinge vorging, so war Becker, der ja bald auch ein Opfer dieser Maßnahmen wurde, dafür wesentlich durch seine „Erklärung und Aufforderung” mit verantwortlich.

Im August 1848 hatte Becker anläßlich einer Versammlung deut-

1 Becker wurde damals auch „chef politique des réfugiés" genannt. Vergleiche dazu ein „laissez passer” für Becker und zwei Genossen, ausgestellt vom Bürgermeister von Hüningen am 8.10.1848, im Nachlaß Becker.

2 J. Ph. Becker, Der Präsident des Wehrbundes "Hilf Dir” an die Mitglieder. Freiheit, Gleichheit, Verbrüderung. Biel, 3. I0.1 848.

s J. B. Hofstetten an J. Ph. Becker, Brief vom I.I 2.I 848, im Nachlaß Becker.

- Als die deutschen Vereine in der Schweiz vom 9.-I I.I 2.I848 in Bern ihren ersten Kongreß abhielten, erkannten sie zwar die Bestrebungen Beckers und des Wehrbundes „Hilf Dir" an. Sie wollten aber als Vereine dem Wehrbund nicht beitreten. Jeder sollte sich selbst entscheiden. Siche: Justiz, Flüchtlinge I848-1895, Kasten 68 d, Mappe: Beschlagnahmte Schriften, Protokolle des ersten Kongresses der deutschen Vereine, abgehalten zu Bern am 9., Io. und I I. Dez. I 848, im Bundesarchiv Bern.

5 Über die Verhältnisse in Deutschland wurde Becker laufend informiert. Vgl. Lommel an J. Ph. Becker, 18.9. 1 848, T. Mögling an Becker, 28.9.1 848, u.a.; alle Briefe im Nachlaß Becker.

- J. Ph. Becker, „Erklärung und Aufforderung”, a. a. O. 
scher Republikaner in Baden erstmals den Gedanken von Schuldscheinen als Form einer Anleihe zugunsten der deutschen Republik entwickelt und war dabei von allen anderen sehr unterstützt worden. Er betonte in seinen Ausführungen, daß gerade der Fehlschlag der vorangegangenen Ereignisse den Beweis dafür liefere, daß man von republikanischer Seite den Finanzangelegenheiten große Aufmerksamkeit zuwenden müsse, um auf jeden Fall erst einmal Geld zu erhalten. Deshalb beschloß man auf dieser Versammlung die Gründung eines Finanzkomitees. Becker wurde in dieses Komitee gewählt und beauftragt, seinen Plan zu verwirklichen. Schon am 2 r. September I 848 erschienen die ersten Probeabdrucke der Schuldscheine, die die Aufschrift „Freiwilliges Anleihen zugunsten der deutschen Republik” und die Unterschrift des Präsidenten des Wehrbundes "Hilf Dir”, Johann Philipp Becker, trugen.1 Eine genaue Beschreibung der Schuldscheine, die der aus Stuttgart gebürtige und in Bern lebende Litograph Kraus lieferte, ${ }^{2}$ finden wir in der „-Evolution”. ${ }^{3}$ Der mißglückte Struveputsch verzögerte das geplante Finanzunternehmen. ${ }^{4}$ Man gründete später ein zweites Komitee, in das Becker ebenfalls wieder gewählt wurde. ${ }^{5}$

Das von Becker geplante Finanzunternehmen sollte einen Teil des benötigten Geldes für die Durchführung der Revolution in Deutschland beschaffen. Becker wandte sich vor allem an diejenigen Freunde der Republik, die nicht so sehr durch persönliche Mitarbeit dem hohen Ziele dienen könnten, die aber gern bereit wären, materielle Opfer zu bringen. Er glaubte, daß jede Zeit, die etwas Neues aufbauen will, das Recht habe, für diese Aufgabe die Zukunft mit zu beanspruchen. Ausgehend von der Tatsache, daß die deutsche Republik einmal kommen müsse, beschlossen die deutschen Republikaner auf Beckers Anraten, zugunsten dieser Republik eine Anleihe zu „kontrahieren” und dafür Schulscheine in verschiedenen Werten auszustellen. ${ }^{6}$

Das Finanzunternehmen war ein öffentliches und unterstand einem

1 Justiz, Flüchtlinge I 848-I 895, Kasten 4I, Mappe: Deutsche Flüchtlinge, Untersuchung gegen die Flüchtlinge Becker, Lommel, Hattemer, Heinzen wegen des rev. Vereins in Biel, Wehrbund „Hilf Dir”, wegen der rev. Zeitschrift „Revolution”, später „Die -Evolution", Bundesarchiv Bern.

- Ruegg, Aus Briefen an J. Ph. Becker, a. a. O., S. 454.

- Die -Evolution, No. 9, S. 3. Fotokopien eines Schuldscheins siehe: Georg Trübner, Johann Philipp Becker - ein Leben für die Freiheit (1809-1 886), (unter Berücksichtigung aller von Becker verfaßten Schriften und Zeitungen, seines Nachlasses in Amsterdam und anderen unveröffentlichten Quellenmaterials, besonders aus Schweizer Archiven), Phil. Habil. Jena 1957, 2 Bände (Maschinenschrift), Band I, S. 127.

- Kern und N. S. von Lueger, Brief an J. Ph. Becker vom 25.1 I.1848, im Nachlaß Becker.

- Die -Evolution, No. 9, S. 3.

- A. a. O., S. 3-4. 
Verwaltungsrat und einer Aufsichtsbehörde. Es wurde ausdrücklich betont, daß der Verwaltungsrat keine Garantie für die Rückzahlung der Gelder übernehmen könne. Die Schuldscheinerwerber müßten den Glauben an die deutsche Republik, die bestimmt einmal kommen werde, haben. Diese deutsche Republik werde ihnen dann die Gelder zurückzahlen. Man wollte keine „Verdummungspolitik” betreiben und vermerkte am Schluß des Schreibens, daß die Rückzahlung immerhin „dem Schicksale und der Zukunft” überlassen bleibe. Dieses denkwürdige Dokument, das uns Aufschluß über die Finanzoperationen der deutschen Emigranten unter Beckers Leitung gibt, stammt vom November i $848 .{ }^{1}$

Der Plan des Finanzunternehmens war bis ins einzelne durchdacht. Es wurden in der Praxis viele solcher Schuldscheine vertrieben und die deutschen Behörden beschwerten sich bei der Schweizer Bundesbehörde, daß sie eine solche Papiergeldausgabe duldete. Besonders der deutsche Flüchtling Karl Daniel Schleher aus Memmingen setzte in Deutschland unter seinen Freunden viele solcher Schuldscheine ab. In Biel dagegen hatte das Unternehmen nur geringe Erfolge. Deshalb bezeichnete auch das Regierungsstatthalteramt in Biel in seinem Bericht vom 13.3.1849 das Geschäft als ziemlich unbedeutend. ${ }^{2}$ So spielerisch uns das Unternehmen im einzelnen heute auch erscheinen mag, so ist es doch ein Beispiel des praktischen Sinnes von Becker, der nie bei der Theorie stehenblieb, der immer bestrebt war, wirkungsvolle Hilfe zu bringen. Dies macht ihn ja gerade zu einem so bedeutenden Praktiker und Organisator der internationalen Arbeiterbewegung.

Becker wurde später wegen angeblich inautorisierter Anfertigung von Banknoten mit einem Kriminalprozeß bedroht. Da sich in den eidgenössischen Behörden viele „Mitschuldige” befanden, gelang es ihm mit Hilfe des Advokaten Emil Vogt, eine Anklage zu verhindern. ${ }^{3}$ Immerhin konnte es Becker zu einer gewissen Genugtuung gereichen, daß sein späterer Freund Mazzini i 850 auf seinen Rat etwas Ähnliches erstrebte. ${ }^{4}$

1 A. a. O., S. 4.

2 Justiz, Flüchtlinge I848-1 895, Kasten 4I, Schreiben vom 13.3.1849, No. 546, Schöni als Regierungsstatthalter in Biel an Direktion der Justiz und Polizei in Bern, Bundesarchiv Bern.

Ruegg, a. a. O., S. 454.

A. a. O., S. 455 . 
BECKERS AGITATORISCHES AUFTRETEN IM NOVEMBER UND DEZEMBER I 848 IN BIEL

Biel blieb weiterhin ein Zentrum für die Republikanisierung Deutschlands. Durch sein Rundschreiben als Präsident des Wehrbundes „Hilf Dir" vom 3.I I.I 848 alarmierte Becker erneut den Schweizer Bundesrat. Becker gab in dem Schreiben einen Überblick über das Legionsund Vereinswesen und forderte alle Mitglieder seines Bundes auf, sich nur an solchen Taten zu beteiligen, durch die der Bund im Auslande nicht an Ansehen verliere. Alle Republikaner, ganz gleich, ob sie schwarz-rot-gold oder rot wären, müßten zunächst ein Teilziel vor Augen haben: die Vernichtung der Fürstengewalt. Erst wenn die Fürsten vernichtet seien, könne man über die Staatsform entscheiden. Gleichzeitig forderte Becker dazu auf, daß sich alle deutsch-republikanischen Elemente im Ausland fest verbinden und stetige Berührung mit den republikanischen Vereinen in Deutschland unterhalten sollten. ${ }^{1}$ Becker wurde in seinem Vorhaben besonders von einer Gruppe deutscher Flüchtlinge in Nancy unterstützt. Diese Kolonne setzte sich aus Mitgliedern zusammen, die sich von Willichs Truppe getrennt hatten. Sie betrachteten Becker als den „erwählten Vertreter aller deutschen Flüchtlinge" und baten ihn deshalb darum, bei der Verteilung der Unterstützungsgelder die neugegründete Kolonne in Nancy nicht zu vergessen. Alle, die in Besançon weggegangen waren, hatten erklärt, daß sie sich von dem selbstherrlichen Willich trennten, nicht aber vom Wehrbund „Hilf Dir”. ${ }^{2}$

Nachdem am 9.11.1848 die Schweiz wegen Verletzung der Neutralität bei den verschiedenen Putschen von der Frankfurter OberPost-Amtszeitung mit Vorwürfen überschüttet worden war, plante die Schweiz strengere Maßnahmen gegen die Flüchtlinge. Dies mußte sich besonders auch gegen Becker richten, der von seinen guten Freunden in den Schweizer Behörden bald unterrichtet wurde. ${ }^{3}$

1 Neitzke, a. a. O., S. 34 .

2 Die deutsche Kolonne zu Nancy an den Bürger J. Phil. Becker in Briel, Präsident des Deutschen Waffenvereins „Hilf Dir”, Brief vom I4.1 I.I848, im Nachlaß Becker.

3 Missivenbuch, No 26 vom 5. Sept. I848-1 5. Juni I 849, S, 130, Schreiben an den Schweizerischen Bundestat, Bern 4.1 2.I 848, Staatsarchiv Bern; Das Polizeidirektorium Bern hat dem Statthalteramt in Biel den Auftrag erteilt, gegen die gefährliche politische Tätigkeit Beckers einzuschreiten, ebenda, S. I 50, Schreiben vom I I.I 2.I 848. Vergleiche dazu auch: Schreiben des Direktors der Justizpolizei Bern, Jäggi, an Regierungsstatthalteramt in Biel vom 4.1 2.1 848: Becker wird beschuldigt, eine dritte Invasion nach Baden vorzubereiten. Das Regierungsstatthalteramt soll die Sache mit Ernst und Umsicht untersuchen. In seinem Gegenschreiben vom 9.I 2.1 848 betonte Regierungsstatthalter Schöni aus Biel, daß niemand eine dritte Invasion vorbereite. Dies wird auch bestätigt durch die 
Becker sandte deshalb am 3. 12.1848 an den Bundesrat in Bern ein Schreiben, ${ }^{1}$ in dem er alle Gerüchte zu widerlegen suchte, die von einem geplanten Einfall seiner Leute nach Deutschland sprachen. Wenn er dabei behauptete, zu allen Zeiten ein Feind von jedem verdeckten Treiben und von allen geheimen Verbindungen gewesen zu sein, so war dies wohl mehr als übertrieben. Er beteuerte weiter, daß er als Präsident des Wehrbundes „Hilf Dir” für Völkerassoziation und Vereinigung aller republikanischen Parteiführer der einzelnen Länder eintreten wolle. Darüber hinaus sei sein Ziel, die Flüchtlingsschaft zu disziplinieren und vor albernen Einfällen zu bewahren. Die Unterstützungsgelder des Bundes zahle man nur an jene Mitglieder, die die Gesellschaft nicht kompromittierten. Sehr diplomatisch betonte er, daß sein Wehrbund der Eidgenossenschaft jederzeit zur Verfügung stehe, falls sie von Feinden bedroht werde. Becker dachte in diesem Zusammenhang besonders an Angriffe von Preußen. Das Schreiben wirkt ziemlich sophistisch, wenn man weiß, daß Becker gerade im Monat Dezember seine Probenummer „Revolution” erscheinen ließ, in der er sehr stark terroristisches Gedankengut vertrat. ${ }^{2}$

Die immer stärker werdende Tätigkeit der Flüchtlinge spaltete die Bevölkerung von Biel in zwei Lager. Die Konservativen versuchten, die revolutionäre Tätigkeit Beckers mit allen Mitteln zu unterbinden. Sie wandten sich am I I.I 2.I 848 an den Bundestat und beklagten sich über die Unruhe, die durch die Wühlerei des "Jungen Deutschlands" und Beckers in ihrer Stadt hervorgerufen wurde. Sie verlangten, daß die Regierung sich besonders gegen das Treiben des Wehrbundes „Hilf Dir" wendete, andernfalls, so drohten sie, werde man dafür sorgen, daß das Land von diesen Gästen befreit werde. Die Adresse trug die Unterschriften von 260 Bieler Bürgern. ${ }^{3}$

Darauf erschien am 27. I 2.I 848 ein Schreiben ganz anderen Inhalts. 13 I Bürger Biels tadelten die Beschwerde an den Bundesrat vom x I. 12.1848 und stellten fest, daß dort Verdächtigungen und Übertreibungen ausgesprochen worden waren, die durch nichts $\mathrm{zu}$ beweisen seien. Besonders nahmen die Unterzeichner Johann Philipp Becker in Schutz. Er habe nirgends seine Pflicht als Schweizer Bürger

Aussageprotokolle vom Großrat Rosel aus Biel, Johann Daniel Schleher aus Memmingen, Großrat Heilmann aus Biel, Martin Benz aus Biel, Ernst Schüler aus Biel und Heinrich Hattemer aus Biel. Alle Schreiben in: Justiz, Flüchtlinge 1848-1895, Kasten 4x, Mappe: Deutsche Flüchtlinge Dez. I 848 bis Jan. I 849 , Akten betreffs J. Ph. Becker und Consorten, Bundesarchiv Bern.

$1 \mathrm{~J}$. Ph. Becker, Schreiben an den Bundesrat in Bern vom 3.1 2.48, in: Justiz, Flüchtlinge 1848-1895, Kasten 41, Mappe Deutsche Flüchtlinge Dez. I848-Jan. 1849, a. a. O.

2 Neitzke, a. a. O., S. 34 .

Justiz, Flüchtlinge I848-I 895, Kasten 4I, Mappe: Untersuchung gegen die Flüchtlinge, Schreiben gegen Becker und die deutschen Flüchtlinge in Biel, No 86, Bundesarchiv Bern. 
vernachlässigt. Im Gegenteil, er habe gerade in den letzten Jahren an den Kämpfen der Schweiz um die so begehrte Freiheit mit sehr viel Aufopferung und Energie teilgenommen, darüber hinaus durch die Gründung einer Zigarrenfabrik in Biel einen neuen Industriezweig entwickelt. Als Literat habe er sich für die Interessen des Schweizer Gewerbewesens überall eingesetzt. ${ }^{1}$

Becker ließ sich auch in der Folgezeit nicht in seiner politischen Tätigkeit einschränken. Mit der Herausgabe der „Revolution”/„Die -Evolution" betrieb er sie sogar noch offener. ${ }^{2}$ Mit der Gründung einer Zeitung verfolgte er das Ziel, in der Schweiz die deutschen Republikaner aller Stände, besonders aber die Vertreter der Arbeiterschaft, fester zusammenzuschließen. Wenn auch die „Revolution” oder - wie sie von der 2. Nummer an genannt wurde - „Die -Evolution" nicht den Siegeslauf nehmen konnte wie später „Der Vorbote”, so zeigte Becker doch gerade durch diese seine erste redaktionelle Tätigkeit im Jahre $1848 / 49$, daß er die publizistischen Fähigkeiten besaß, um noch größere Aufgaben zu lösen.

In seiner ersten Ausgabe vom r.I2.I848 formulierte Becker die Hauptaufgaben der „Revolution” wie folgt:

„Unerbittlicher Kampf gegen das Fürstentum, seine Repräsentanten und Lakaien. Vernichtung der Pfaffengewalt und Verbreitung einer vernünftigen Weltanschauung. Entschiedene Vertretung der Interessen der sogenannten unteren Volksklassen. Bekämpfung der Nationalvorurteile, wo wir sie treffen mögen, Verbreitung der Idee einer Völkerassoziation, einer brüderlichen Vereinigung der republikanischen Parteiführer aller Länder."3

Alle Artikel waren leicht verständlich geschrieben. Es wurde hier keine Zeitung für Philister am Biertisch redigiert, die mit olympischer Ruhe die Weltereignisse in weiter Ferne betrachteten. Es sollte vielmehr eine Zeitung in die Welt hinausgehen, die mit aller Leidenschaftlichkeit, mit aller Liebe und mit allem $\mathrm{Haß}$ geschrieben war, die - und das war für Becker das Wichtigste - auf jeder Zeile eine klare Parteinahme für die Ausgebeuteten erkennen ließ. Diese Parteinahme im Interesse der schaffenden Menschen ist zu allen Zeiten ein charakteristisches Merkmal der Beckerschen journalistischen Tätigkeit geblieben. ${ }^{4}$

1 Justiz, Flüchtlinge I 848-1895, Kasten 41, Mappe: Untersuchung gegen die Flüchtlinge, Schreiben der Bieler Einwohner vom 27.1 2.1 848 an den Bundesrat in Bern, Bundesarchiv Bern.

2 Die Revolution, ein politisches Wochenblatt, Organ der Gesellschaft „Hilf Dir”, Biel I 848, Freitag I.I 2. I848, Redakteur und Verleger J. Ph. Becker (unter diesem Titel erschien nur ein Probeblatt); Die -Evolution, ein politisches Wochenblatt, verantwortlicher Redakteur J. Ph. Becker, Biel $1848 / 49$.

Die Revolution, S. r.

- Die Revolution, S. I-2. 
Als am I.I 2.I 848 ein Probeblatt dieser Zeitung in Biel erschien, erregte es ganz besonders den Ärger des politischen Departements. An der Spitze dieser Abteilung stand Dr. Furrer, der in seinem Bericht an den Bundesrat schrieb, daß dieses Unternehmen allem bisherigen Treiben der Fremden in der Schweiz die Krone aufsetze ${ }^{1}$. $\mathrm{Da}$ der Bundesrat das weitere Erscheinen des Blattes untersagte, wählte Becker deshalb den milderen Titel „Die -Evolution” für seine Zeitung. Von ihr liegen vom 5.I.I 849 bis zum 30.3.1 849 elf Nummern vor, dazu kommt ein Probeblatt vom 29.I 2.I 848. Da das Erscheinen der ,-Evolution” zeitlich schon über den diesem Artikel gesteckten Zeitraum hinausgeht, darf ich mich mit diesen Hinweisen begnügen.

\section{VI}

\section{ZUSAMMENFASSUNG}

Wenn man die Tätigkeit Beckers 1848 in Biel zusammenfassend beurteilt, so muß man feststellen, daß er die schöne Stadt am Fuße des Schweizer Jura tatsächlich zu einem revolutionären Zentrum deutscher Flüchtlinge machte. Becker bekannte sich im Revolutionsjahr 1848 nicht nur in Worten und Aufrufen zu der von ihm so heiß herbeige'sehnten Republik, er ließ es auch vor allem nicht an Taten fehlen, um der Verwirklichung dieses Zieles zu dienen. Ohne Rücksicht auf persönliche Nachteile - er hatte sich in Biel gerade eine Existenz geschaffen - nahm er jede Möglichkeit wahr, sich für die erstrebte deutsche Republik einzusetzen. Als besonders günstig erwies es sich, daß Becker bis 1847 aktiv am Kampfe für die Erringung demokratischer Ziele in der Schweiz teilgenommen hatte und sich deshalb bei einem großen Teile der Bieler Bevölkerung Achtung und Anerkennung erworben hatte. Andererseits kam Becker auch bei vielen der hier angeführten Handlungen zugute, daß die Schweizer Behörden den politischen Flüchtlingen gegenüber eine gewisse Nachsicht übten und ihr Asylrecht hochhielten.

In Biel ist Becker durch seine Hilfsbereitschaft, sein Organisationstalent, seine ständige Einsatzbereitschaft und seine große Begeisterungsfähigkeit für die Sache der Revolution zu der markantesten Persönlichkeit unter den deutschen Republikanern in der Schweiz herangereift. Am bedeutendsten erscheint mir dabei Beckers Tätigkeit 1848 in Biel in seiner Eigenschaft als Leiter des „Zentralausschusses

1 Flüchtlinge, Justiz 1848-1895, Kasten 4I, Schreiben Dr. Furrers im Auftrage des politischen Departements an den eidgenössischen Bundesrat, Zürich, den I 2. I 2.I 848, A I07, Bundesarchiv Bern. 
der Deutschen aus der Schweiz" und seine Tätigkeit als Präsident im Wehrbund „Hilf Dir” gewesen zu sein. Auf Grund der in dieser Eigenschaft veröffentlichten „Instruktion” und dem „Rundschreiben” wurde Becker dann angeklagt und mußte nach Bestätigung des Urteils durch das Obergericht in Bern ${ }^{1}$ am 26.2.1849 seine „Bannisation" antreten und die Schweiz verlassen.

Die Erfahrungen aber, die Becker I 848 durch seine Bieler Tätigkeit auf den verschiedensten Gebieten sammeln konnte, haben zweifellos dazu beigetragen, daß er sich 1849 in der Badisch-Pfälzischen Revolution als Truppenführer so auszeichnete, daß er später von seinem Freunde Friedrich Engels als der ,einzige deutsche Revolutionsgeneral"2 bezeichnet wurde.

\footnotetext{
1 Das Regierungsstatthalteramt in Biel (Schöni) an die Direktion der Justiz und Polizei des Kanton Bern, Schreiben vom 8.3.1849, No. 546, in: Justiz, Flüchtlinge I848-1895, Kasten 4I, Bundesarchiv Bern.

${ }^{2}$ Friedrich Engels, Dem Gedächtnis Johann Philipp Beckers, in: Der Sozialdemokrat, Organ der Sozialdemokratie deutscher Zunge, Zürich, 17.12.1886, No 51, Titelseite. Über Beckers Tätigkeit in der Badisch-Pfälzischen Revolution, vergleiche auch: Georg Trübner, Johann Philipp Becker - ein Leben für die Freiheit, Band 1, S. I60-220.
} 